\title{
Einladung zum Lebkuchenkongress 2018
}

Liebe Kolleginnen und Kollegen,

der 47. Fortbildungskongress für Assistenzpersonal in der Radiologie, Nuklearmedizin und Strahlentherapie in Nürnberg wird dieses Jahr wieder wie gewohnt am zweiten Advent-Wochenende stattfinden. Der Tagungsort Meistersingerhalle ist sehr zentral gelegen, so dass er mit den öffentlichen Verkehrsmitteln gut erreichbar ist. Für die, die mit dem Auto anreisen, stehen ausreichend Parkplätze zur Verfügung. Nach dem Vermehren von Wissen können sie einen Spaziergang mit Glühwein und Lebkuchen über den schönen Christkindlesmarkt machen.

Der diesjährige Schwerpunkt in der Röntgendiagnostik ist am Freitag das Knie mit Anatomie, Indikationen, Einstelltechnik, Scan-Protokollen und typischen Befunden. Und ein weiteres Thema ist die oft unterschätzte Bedeutung der Röntgen-Bettlunge und aktuelle Hygieneanforderungen.

Am Samstagnachmittag informieren wir sie über die neue Strahlenschutzverordnung und praktische Strahlenschutzmaßnahmen für Patient und Personal.

In der Nuklearmedizin geht es um bewährte Diagnostik wie die Nieren- und Lungenszintigrafie, SPECT- und PET Untersuchungen, aber auch um Strahlenschutz und QM. Interessant ist auf jeden Fall auch der Vortrag „Radiopeptidtherapie mit Lutathera in die klinische Zulassung“.

In der Strahlentherapie ist am Freitag das Haupthemen „Lungenkarzinom“ und am Samstagvormittag „Mammakarzinom“

Für die MTRA-Schüler gibt es traditionell wieder ein speziell auf sie zugeschnittenes Programm, an dem sie selbstverständlich kostenlos teilnehmen können. Wichtig: eine vorherige Anmeldung ist unbedingt nötig.

Ergänzt werden die fachorientierten Vorträge durch Praktika in den Klinikseminaren, die am Samstagvormittag im Klinikum Nürnberg-Nord und -Süd angeboten werden. In den Lunch-Symposien am Freitag und Samstag erhalten sie neben einem
„Verpflegungspaket“ aktuelle Informationen aus der Industrie.

Zum letzten Mal besteht auf diesem Kongress die Möglichkeit die Fachkunde und Kenntnisse nach beiden Verordnungen (RöV undStrISchV) zu aktualisieren, da ab 31.12.2018 eine neue Strahlenschutzverordnung für alle in Kraft tritt. Auch wir sind gespannt, ob ab nächstem Jahr die Kursstruktur geändert werden muss.
Wir freuen uns auf Sie!

Prof. Dr. Dr. R. Loose

Katja Röhr

Dr. rer. nat. M. Wucherer

Weitere Informationen zum Programm und zur Anmeldung finden Sie auf www.lebkuchenkongress.de. 


\section{Premiere auf dem Lebkuchenkongress: Zertifizierungskurs „FIR-Modul 3“}

Sehr geehrte Kolleginnen und Kollegen,

die Häufigkeit und auch die Komplexität Interventionell-radiologischer Eingriffe nimmt stetig zu, womit eine atraumatischere und schonendere Behandlung vieler Patienten möglich wird. Das Klinikum Nürnberg bietet in Kooperation mit der VMTB, der DeGIR und DRG zum diesjährigen Lebkuchenko- gress erstmals einen zertifizierten Kurs „Fachkraft Interventionelle Radiologie (FIR)“, Modul 3 an. Dieser Kurs richtet sich an alle, die in der interventionellen Radiologie tätig sind bzw. dies in Zukunft sein werden. Im kompakten Format vertiefen Sie Ihre Kenntnisse im Komplikationsmanagement und der Qualitätssicherung, sowie auf spezifischen interventionellen Feldern (abdominelle, neuroradiologische, sowie Mamma Interventionen). Der Kurs endet mit einer Prüfung und diejenigen Teilnehmer, die bereits Modul 1 und 2 erfolgreich absolviert haben, können damit das VMTB-DeGIR-DRG Zertifikat erlangen.
Wir freuen uns, Sie im vorweihnachtlichen Nürnberg begrüßen zu dürfen.

Ihre

K. Roehr und Prof. Dr. M. Lell

Weitere Informationen zum Zertifizierungskurs erhalten Sie auf www.lebkuchenkongress.de $>$ Programm > Zertifizierungskurs „FIR-Modul 3“ 